\title{
Lesiones subepicárdicas en Chile (Infarto Agudo del Miocardio con supradesnivel del Segmento ST). Experiencia con Telemedicina
}

\author{
Drs. Edgardo Escobar C., Margarita Véjar J., Roberto del Pino. \\ Internos de Medicina: Pablo González, Ricardo Escobar \\ ITMS Telemedicina de Chile
}

\section{Resumen}

Antecedentes: La transmisión telefónica del electrocardiograma (ECG) a un centro de análisis ha permitido el diagnóstico precoz del infarto agudo del miocardio (IAM), incluso en lugares remotos de nuestro país. La ley AUGE exige el tratamiento inmediato del IAM con trombolisis y/o el traslado del paciente a un centro de referencia para su ulterior tratamiento.

Objetivo: Tabular las características demográficas, distribución geográfica de la población estudiada, fecha y hora de presentación del IAM.

Métodos: De un total de 523.371 ECG se analizaron 3.656 trazados con lesiones subepicárdicas definidas por desnivel superior de ST de a lo menos de $1 \mathrm{~mm}$ en dos o más derivaciones consecutivas (IAM con SDST). LoS ECG fueron adquiridos con sistemas Aerotel o Cardiette y analizados por dos cardiólogos. Resultados: Se confirmó la mayor incidencia de IAM en hombres (69.2\% vs 30.8\%) y la presentación del IAM a una edad mayor en mujeres. Se registró un mayor número de IAM entre los meses de Abril y Julio, y entre las 09 y 13 horas. Los tiempos de respuesta del sistema desde el momento de la recepción del ECG hasta el envío del informe no superó los $10 \mathrm{~min}$, a cualquier hora del día o la noche, tanto en días hábiles como no hábiles. Conclusión: Se consignaron antecedentes epidemiológicos del IAM en el país, a la vez que se comprobó que el sistema permite un diagnóstico precoz del IAM, lo cual puede tener un impacto positivo en el tratamiento de esta patología.

Palabras claves: Electrocardiograma, Infarto agudo del miocardio.

\section{Telephonic transmission of electrocardiograms for early diagnosis of ST elevation Acute Myocardial Infarction in Chile}

Background: Telephonic transmission of an electrocardiogram (ECG) from rural areas to a center with cardiologist in place to analyze the records has been recently incorporated in Chile, allowing earlier diagnosis of an Acute Myocardial Infarction, even in remote places. Such early diagnosis should prompt faster thrombolysis or primary angioplasty.

Aim: To describe demographics of the population in which diagnostic ECG were taken, plus date and time of the day when the diagnosis of AMI was made.

Correspondencia: Dr. Edgardo Escobar

Callao 3341

Fax 4374943

Correo Electrónico: e.escobar@itms.cl 
E. Escobar, M. Véjar, R. del Pino.

Method: Out of 523.371 ECG, we identified 3.656 records with epicardial lesions, defined as ST elevation of at least $1 \mathrm{~mm}$ in 2 concordant leads (STEAMI). The records were obtained with systems Aerotel or Cardiette, and were analyzed by 2 cardiologists.

Results: We found higher incidence of STEAMI in man (69.2\% vs 30.8\%). Older age was more frequently seen in women with STEAMI. A higher incidence of STEAMI was found between April and June, being more frequently observed between 9 AM and 13 PM. The delay between the reception of the ECG and its interpretation was no longer than $10 \mathrm{~min}$, regardless the time of the day, and including holidays

Conclusion: We confirmed some epidemiological data regarding STEAMI in Chile. This technology allows the early diagnosis of the STEAMI from remotes areas of our country.

Key words: Electrocardiogram, acute myocardial infarction.

Recibido el 2 de febrero de 2009. Aceptado el 13 de febrero de 2009

Rev Chil Cardiol 2009; 28: 73-80

\section{Introducción}

El diagnóstico precoz del Infarto Agudo del Miocardio (IAM) se ha impuesto como un objetivo fundamental para realizar su tratamiento oportuno con angioplastía primaria o trombolisis 1 .

En el año 2004 el Ministerio de Salud (MINSAL) implementó la ley AUGE (actualmente GES) para el diagnóstico del IAM y con la asesoría de la Sociedad Chilena de Cardiología y Cirugía Cardiovascular confeccionó un Protocolo de Tratamiento. La ley AUGE exige el tratamiento inmediato el IAM para realizar trombolisis y/o trasladar al paciente a un centro de referencia para su ulterior tratamiento2.

Si bien el diagnóstico del IAM se basa en la triada de cuadro clínico, electrocardiograma (ECG) y enzimas, es el ECG la piedra angular del diagnóstico, el que deber ser adquirido en los primeros 15 minutos de iniciado el dolor torácico.

La mejor respuesta a este requerimiento esta Teleelectrocardiografía que permite la trasmisión telefónica del ECG a un centro de análisis incluso desde lugares muy remotos. Por dicha razón el MINSAL el 1 de Mayo del 2004 identificó al servicio ITMS, Telemedicina de Chile, como el soporte del diagnóstico electrocardiográfico del IAM, para poder realizar trombolisis las 24 horas del día y los 365 días del año.

El objetivo del presente trabajo es analizar las características demográficas y distribución geográfica, de la población estudiada, fecha y horas de presentación del IAM.

\section{Método}

Se analizaron un total de 523. 371 ECG adquiridos con equipos Aerotel HP o Cardiette, trasmitidos telefónicamente a un centro de llamados de Telelemedicina donde fueron analizados utilizando un sistema computacional. El detalle de la tecnología y la comparación con los ECG adquiridos con equipos tradicionales ha sido comunicada previamente ${ }^{3}$.

Se debe destacar que aplicando el método de Altman y Bland 4 los trazados adquiridos con el método tradicional son absolutamente comparables a los registrados por Telemedicina. En la Unidad Coronaria del Hospital Salvador se compararon, los trazados de diez pacientes con evento coronario agudo sin encontrarse ninguna diferencia entre el ECG adquirido con los equipos mencionados y el registrado con equipo tradicional 5 .

El tiempo máximo de envío del informe al lugar determinado, para estos casos, es de diez minutos según protocolo. Este tiempo generalmente es más breve, tanto en horario diurno como nocturno y en días hábiles y no hábiles.

En la gran mayoría de estos casos se logra interactuar con el médico tratante 0 , en su defecto, con enfermera o paramédico. El sistema de Telemedicina sólo es responsable del diagnóstico electrocardiográfico 
y no tiene capacidad de decisión sobre el tratamiento ni el seguimiento de los pacientes

La figura 1 esquematiza la forma de funcionamiento del sistema.

EI IAM con SDST fue definido por un desnivel superior de ST de a lo menos $1 \mathrm{~mm}$ en dos o más derivaciones contiguas, desestimándose alteraciones ambiguas, según opinión de dos observadores.
Solamente se analizó un ECG por paciente, a menos que se presentaran lesiones en otras derivaciones electrocardiográficas a la analizada previamente.

La figura 2 muestra un ejemplo típíco de lesión subepicárdica tal como se obtiene con el sistema descrito. Se hizo un estudio de la distribución de los trazados con lesión por región del país y porcentajes por sexo y edad.

\section{Servicio de Tele-ECG en apoyo a Eventos Coronarios Agudos}

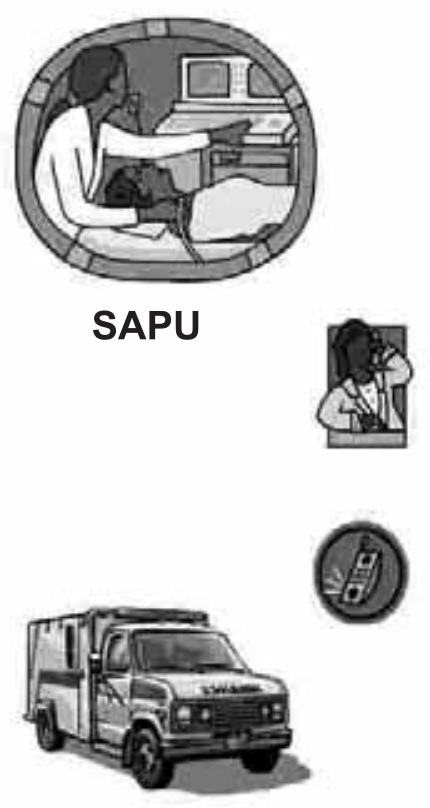

SAMU
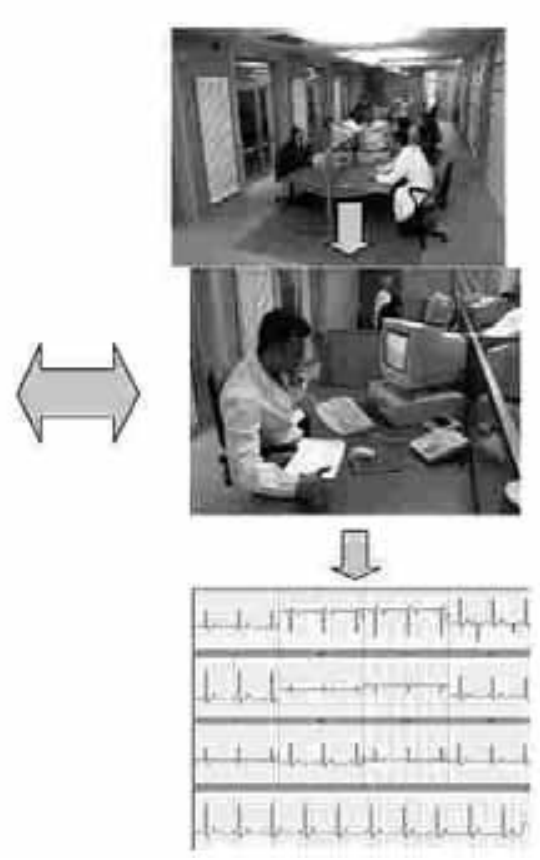
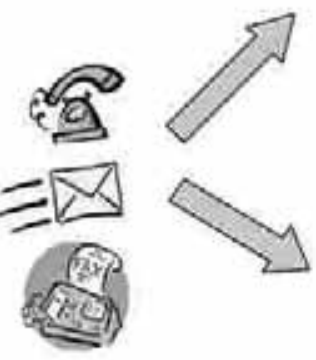

\section{Hospital de} Referencia

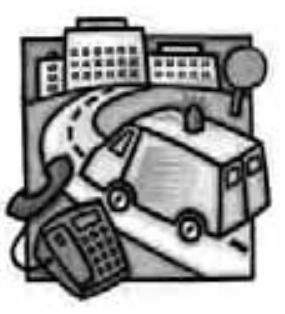

SAPU o Traslado

Figura 1. Flujograma de Teleelectrocardiografía. EL ECG es transmitido telefónicamente desde el Servicio de Urgencia o ambulancia a la base de informes y simultáneamente al Centro de Referencia. 
E. Escobar, M. Véjar, R. del Pino.

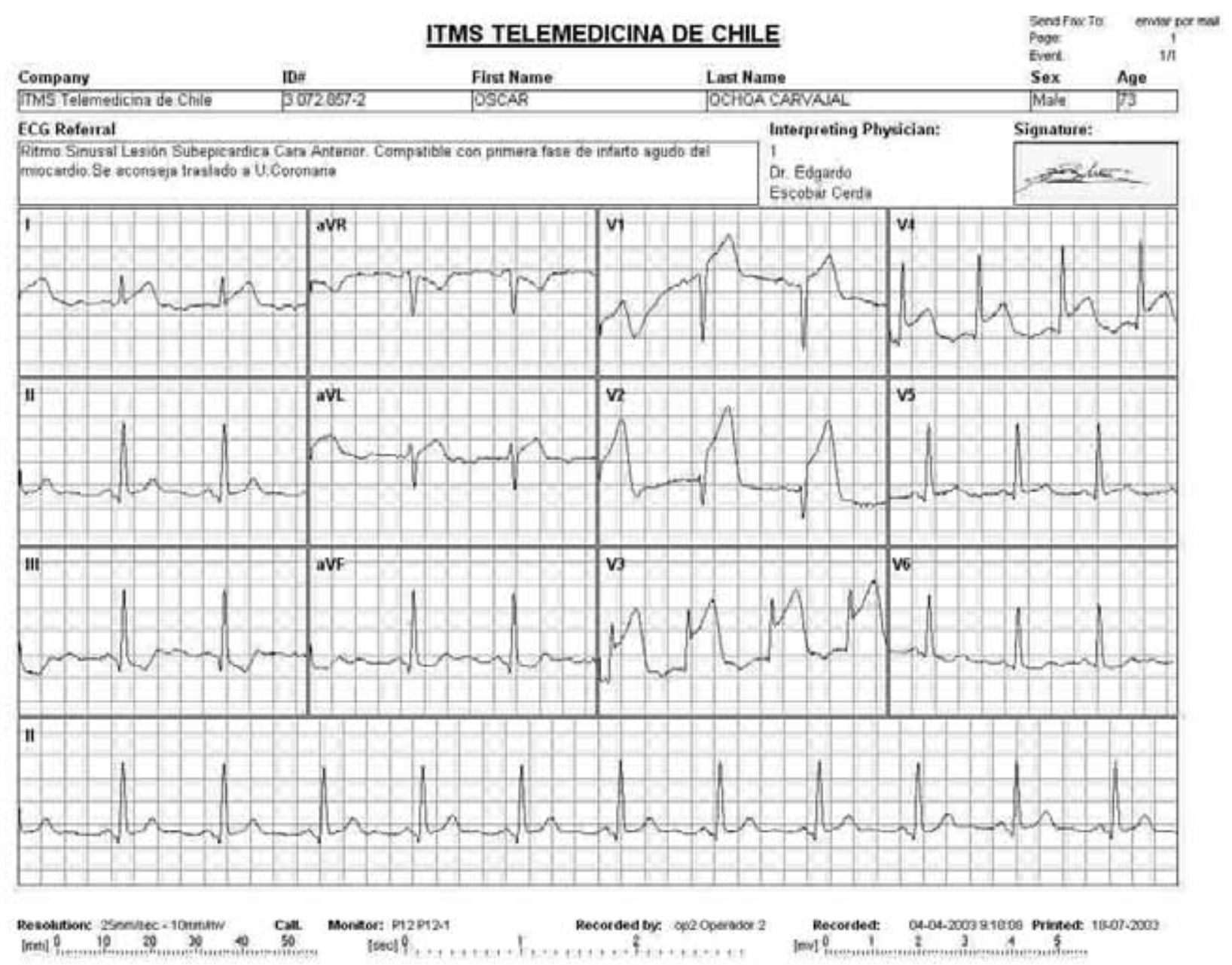

Figura 2. Ejemplo de lesión subepicárdica de cara anterior tal como se registra con el sistema de Telemedicina.

\section{Resultados}

La distribución por edades y por región del país se presenta en la Tabla 1.

En la Tabla 2 se muestra la distribución por sexo y por región. La incidencia de IAM es mayor en hombres $(69.2 \%$ vs $30.8 \%)$.

La presentación del IAM en las mujeres es a edad más tardía que en los hombres.

Se registró un mayor número de IAM entre los meses de Abril y Julio, y entre las 09 y 18 horas (Figura 3). Los tiempos de respuesta del sistema desde el momento de la recepción del ECG hasta el envío del informe no superó los $10 \mathrm{~min}$, a cualquier hora del día o de la noche, tanto en días hábiles como no hábiles. 
Tabla 1. Distribución por edades y región del país

\begin{tabular}{lcccccccccc}
\hline REGION & $\mathbf{1 0 - 1 9}$ & $\mathbf{2 0 - 2 9}$ & $\mathbf{3 0 - 3 9}$ & $\mathbf{4 0 - 4 9}$ & $\mathbf{5 0 - 5 9}$ & $\mathbf{6 0 - 6 9}$ & $\mathbf{7 0 - 7 9}$ & $\mathbf{8 0 - 8 9}$ & $\mathbf{9 0 - 9 9}$ & $\begin{array}{c}\text { Total } \\
\text { general }\end{array}$ \\
\hline 1 & $0 \%$ & $9 \%$ & $9 \%$ & $5 \%$ & $27 \%$ & $23 \%$ & $23 \%$ & $5 \%$ & $0 \%$ & 22 \\
2 & $0 \%$ & $6 \%$ & $0 \%$ & $21 \%$ & $31 \%$ & $19 \%$ & $15 \%$ & $8 \%$ & $0 \%$ & 52 \\
3 & $0 \%$ & $0 \%$ & $6 \%$ & $15 \%$ & $22 \%$ & $26 \%$ & $22 \%$ & $9 \%$ & $0 \%$ & 65 \\
4 & $0 \%$ & $2 \%$ & $3 \%$ & $12 \%$ & $22 \%$ & $21 \%$ & $21 \%$ & $12 \%$ & $1 \%$ & 165 \\
5 & $0 \%$ & $1 \%$ & $4 \%$ & $11 \%$ & $23 \%$ & $23 \%$ & $23 \%$ & $12 \%$ & $2 \%$ & 446 \\
6 & $0 \%$ & $2 \%$ & $4 \%$ & $10 \%$ & $14 \%$ & $26 \%$ & $30 \%$ & $11 \%$ & $2 \%$ & 266 \\
7 & $0 \%$ & $1 \%$ & $3 \%$ & $9 \%$ & $17 \%$ & $20 \%$ & $33 \%$ & $14 \%$ & $3 \%$ & 206 \\
8 & $0 \%$ & $1 \%$ & $3 \%$ & $10 \%$ & $22 \%$ & $27 \%$ & $22 \%$ & $14 \%$ & $1 \%$ & 484 \\
9 & $0 \%$ & $0 \%$ & $2 \%$ & $11 \%$ & $20 \%$ & $30 \%$ & $22 \%$ & $13 \%$ & $2 \%$ & 288 \\
10 & $1 \%$ & $1 \%$ & $6 \%$ & $11 \%$ & $19 \%$ & $30 \%$ & $20 \%$ & $10 \%$ & $2 \%$ & 319 \\
11 & $9 \%$ & $0 \%$ & $9 \%$ & $9 \%$ & $36 \%$ & $18 \%$ & $9 \%$ & $9 \%$ & $0 \%$ & 11 \\
12 & $0 \%$ & $0 \%$ & $0 \%$ & $10 \%$ & $30 \%$ & $40 \%$ & $20 \%$ & $0 \%$ & $0 \%$ & 10 \\
13 & $0 \%$ & $2 \%$ & $4 \%$ & $11 \%$ & $26 \%$ & $25 \%$ & $23 \%$ & $9 \%$ & $1 \%$ & 1322 \\
Total general & $\mathbf{0} \%$ & $\mathbf{1} \%$ & $\mathbf{4} \%$ & $\mathbf{1 1} \%$ & $\mathbf{2 2} \%$ & $\mathbf{2 4} \%$ & $\mathbf{2 3} \%$ & $\mathbf{1 1 \%}$ & $\mathbf{2 \%}$ & $\mathbf{3 6 5 6}$ \\
\hline
\end{tabular}

Tabla 2. Distribución por sexo y por región del país

\begin{tabular}{rccc}
\hline REGION & Femenino & Masculino & Total general \\
\hline 1 & 8 & 14 & 22 \\
2 & 10 & 42 & 52 \\
3 & 13 & 52 & 65 \\
4 & 50 & 115 & 165 \\
5 & 147 & 299 & 446 \\
6 & 79 & 187 & 266 \\
7 & 65 & 141 & 206 \\
8 & 166 & 318 & 484 \\
9 & 77 & 211 & 288 \\
10 & 118 & 201 & 319 \\
11 & 2 & 9 & 11 \\
12 & 5 & 5 & 10 \\
13 & 433 & 889 & 1322 \\
Total & & & 3656
\end{tabular}



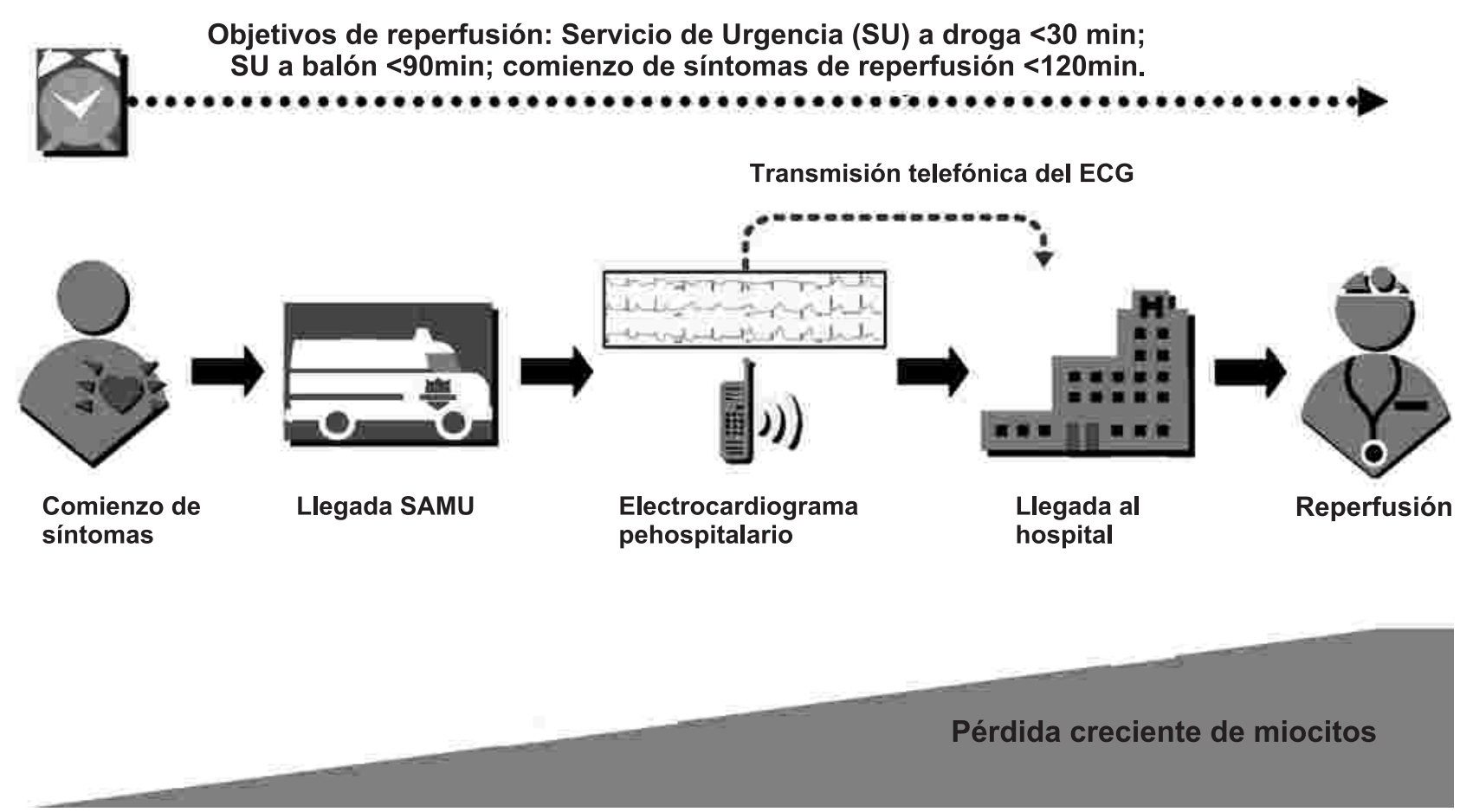

Figura 3. Adaptada de Ting $\mathrm{H}$ et al ${ }^{9}$ destacando la pérdida creciente de miocitos desde el momento de la consulta y los tiempos ideales de acceso al tratamiento.

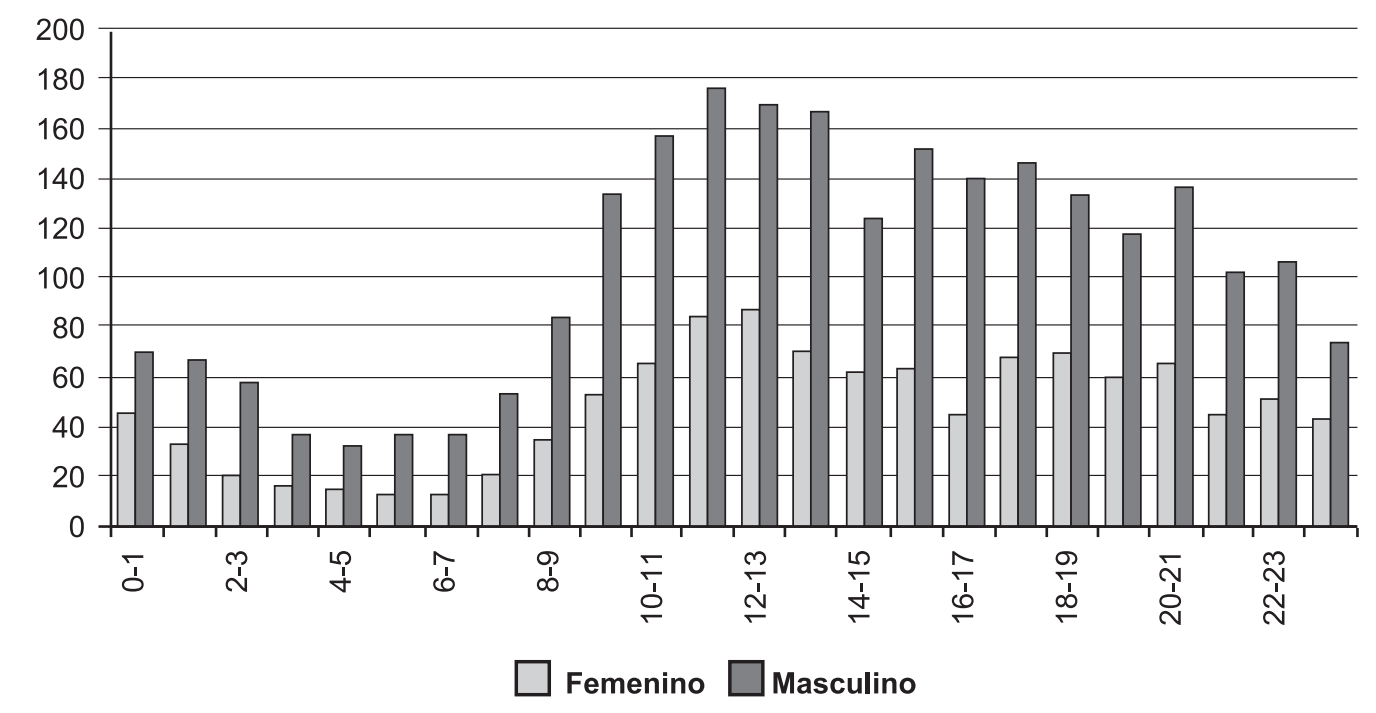

Figura 4. Distribución de lesiones subepicárdicas por horario y sexo 


\section{Discusión}

Hay distintas maneras de interpretar el ECG, una vez obtenido en lugares donde no se encuentran profesionales calificados para el efecto, siendo tres las principales: a) algoritmos computacionales integrados al equipo de registro, b) interpretación por un paramédico entrenado y c) transmisión telefónica del registro a un centro de llamados que cuente con médicos acreditados para el informe.

Los dos primeros están sujetos a errores de interpretación por lo que el sistema utilizado en Chile de transmisión telefónica es sin duda el más adecuado. Este último representa un costo menor ya que los equipos de registro son simples, no necesitan papel ni requieren mantención. Sólo requiere tener línea telefónica fija o móvil y un fax o correo electrónico. Tiene además la enorme ventaja de interacción entre el médico de atención primaria y el médico especialista que está disponible en turno continuado las 24 horas del día, todos los días del año.

La diversidad de los sistemas de atención, el diferente tamaño de las ciudades y los sistemas de comunicación disponibles plantean diferentes tipos de solución pero lo básico es activar el protocolo lo más precozmente posible. Para el paciente, el tiempo entre el primer contacto médico y la trombolisis o la angioplastía primaria es una medida relevante de la calidad de atención.

La posibilidad de realizar un ECG prehospitalario contribuye sin duda a mejorar la atención de pacientes con IAM con SDST, ya que permite la realización de trombolisis oportuna y disminuir el tiempo de acceso a una angioplastía coronaria.

Scalvini et al. ${ }^{6}$, han demostrado en Italia la disminución de 43 min en el tiempo de traslado de los pacientes a una Unidad Coronaria, utilizando el sistema de Teleelectrocardiografía con respecto al sistema público italiano tradicional. Otros autores han demostrado una disminución del tiempo "puertadroga" de 10 min y reducción de 15-20 min en tiempo "puerta-balón" con la implementación de ECG prehospitalario 7,8 .

Las guías de la American Heart Association (AHA $)^{9}$ recomiendan la adquisición y uso de un ECG prehospitalario en el Servicio de Urgencia en pacientes en los que sospecha un evento coronario agudo (ECA). La recomendación actual es clase $2 a$ con un nivel de evidencia B. La figura 4, adaptada de esta publicación, esquematiza los tiempos ideales a conseguir en el manejo de esta situación.

La importancia de aplicar estas recomendaciones ha sido evaluada recientemente 10 y confirmado claramente su utilidad, aumentando el uso y la rapidez de técnicas de reperfusión, con tendencia a un menor riesgo de mortalidad.

Sin duda lo anterior tiene que formar parte de un protocolo para cumplir el objetivo central que es el tratamiento oportuno de un ECA, especialmente de un IAM. EI Ministerio de Salud de Chile, con el apoyo de la Sociedad Chilena de Cardiología y Ciru-gía Cardiovascular, confeccionó un protocolo según exigencias de la ley AUGE, de "Manejo de pacientes con Infarto agudo del Miocardio con elevación del segmento ST", que incluye en sus algoritmos un ECG informado por Telemedicina en hospitales tipo 3 y $4^{2}$, así como un sistema de referencia de los pacientes una vez que se haya realizado, o para que se efectúe la trombolisis, o idealmente, una angioplastía primaria.

Esto ha significado romper cierta resistencia inicial y ha presentado un desafío que, paulatinamente, está siendo superado y se ha traducido en una disminución de la mortalidad por IAM de un 12 a un $8,6 \%{ }^{11}$.

El sistema de telemedicina ha contribuido por otro lado a evitar traslados innecesarios en casos en que se ha sospechado un ECA en los cuales el ECG es totalmente normal inicialmente y en un control posterior, lo que ha contribuido a mejorar la eficiencia de los sistemas de traslado a nivel de la atención primaria ${ }^{12-15}$.

\section{Limitaciones}

EI número de IAM presentado no representa la totalidad de casos que se han presentado en el país ya que no toda institución de salud ni todos los servicios de urgencia están adscritos al sistema de Telemedicina. Sin embargo, sí lo están los servicios de urgencia dependientes del Ministerio (SAPU), 
por lo que la muestra es representativa y permite una adecuada evaluación del sistema público. El sistema de Telemedicina sólo es responsable del diagnóstico electrocardiográfico. Las decisiones terapéuticas son tomadas por los médicos tratantes según protocolo, por lo que no tenemos datos sobre el porcentaje de casos en que se realizó trombolisis o angioplastía primaria, ni sobre el seguimiento de estos pacientes.

\section{Conclusiones}

Los datos aquí presentados no difieren de lo descrito en la literatura, en cuanto a distribución horaria, sexo y edad, del IAM con SDST.
El sistema de Telemedicina constituye un apoyo muy importante para la atención primaria, supliendo la falta de especialistas. Ha contribuido a hacer posible la implementación de la ley AUGE en cuanto al tratamiento del IAM se refiere, y colabora en el diagnóstico y tratamiento de otras emergencias cardiovasculares.

\section{Agradecimientos}

Drs. Patricia Adriazola, Arturo Arribada, Walter Kuhne, José de Horta, Guillermo de la Cuadra, Miguel Benedikt, Enrique Almagro, Patricio Castro, Rafael Méndez, Francesca Bello, Médicos de turno de Telemedicina.

\section{Referencias}

1. ANTMAN EM, HAND M, ARMSTRONG PW, BATES ER, GREEN LA, HALASYAMANI LK, et al. ACC/AHA guidelines for the management of patients with ST elevation myocardial infarction. Circulation 2008; 117: 296-329.

2. Protocolo AUGE. Manejo de pacientes con Infarto Agudo del miocardio con elevación del segmento ST. Minsal, Abril 2004.

3. ESCOBAR E, ADRIAZOLA P, CASTRO P, MONTANER R, MENDEZ R, ROSSEL V, et al. Teleelectrocardiografía . Experiência Preliminar. XL Congreso Chileno de Cardiologia y Cirugía Cardiovascular, Rev Chil Cardiol 2003, 22: 125.

4. ALTMAN DG, BLAND JM. Measurement in Medicine: the analysis of method comparison studies. The Statitian 1983; 32: 307-317.

5. ROSSEL V. Comunicación personal.

6. SCALVINI S, ZANELLI E, CONTI C. Assessment of prehospital chest pain using telecardiology. Journal of Telemedicine and Telecare 2002; 8: 231-236.

7. CANTO JG, ROGERS WJ, BOWLBY IJ, FRENCH WJ, PEARCE DJ, WEAVER WD. The prehospital electrocardiogram in acute myocardial infraction: is its full potential being realized? National Registry of myocardial infarction 2 investigators. J Am Coll Cardiol 1997; 29: 498-505.

8. CURTIS JP, PORTNAY EL, WANG Y, MCNAMARA RL, HERRIN J, BRADLEY EH, et al. The prehospital electrocardiogram and time to reperfusion in patients with acute myocardial infraction, 2000-2002: findings from the National Registry of myocardial infarction-4. J Am Coll Cardiol 2006; 47: 1544-1552.

9. TING HH, KRUMHOLZ HM, BRADLEY EH, CONE DC, CURTIS JP, DREW BJ, et al. Implementation and Integration of Prehospital ECGs into systems of care for acute coronary syndrome. Scientific Statement from the American heart Association. Circulation 2008; 118: 1066-1079.

10. DIERCKS DB, CONTOS C, CHEN AY, POLLACK CH, WIVIOTT SD, RUMSFELD JS, et al. Utilization and impact of Prehospital Electrocardiograms for patients with acute ST-segment elevation Myocardial infarction. J Am Coll Cardiol 2009; 53: 161-166.

11. CAMPOS $P$, NAZZAL C, SANHUEZA $P$, CORBALÁN R, BARTOLUCCI J, LANAS F, et al. Impacto del plan AUGE en el tratamiento de pacientes con Infarto agudo del

Miocardio con SDST en hospitales chilenos. Rev. Chil Cardiol. 2007; 26: 254.

12. VILLARROEL R. Estudio de eventos coronarios en Hospital La Ligua. Octubre 2003-Mayo 2004) basado en informes de Telemedicina de Chile. Comunicación personal.

13. MOLINARI G, REBOA G, FRASCIO M, LEONCINI M, ROLANDI A, BALZAN C, et al. The role of telecardiology in supporting the decision- making process of general practioners during the management of patients with suspected cardiac event. Journal of Telemedicine and Telecare 2002; 8: 97-101.

14. MOLINARI G, VALBUSA A, TERRIZZANO M, BAZZANO M, TORELLI L, GIRARDI N. Nine years' experience of telecardiology in primary care. Journal of Telemedicine and Telecare 2004; 10: 249-253.

15. ESCOBAR E. Teleectrocardiography in Chile: impact on health care system. Minutes XV World Congress of Cardiology. Buenos Aires,Argentina, Mayo 2008. 